\title{
Special issue: Micro and Nano Flows (MNF2014)
}

\author{
Carola König $^{1}$ - Alberto Redaelli ${ }^{2}$ - Dimitris Drikakis ${ }^{3}$
}

Published online: 20 October 2015

(C) Springer-Verlag Berlin Heidelberg 2015

There is an increasing interest in understanding heat and fluid flow on small scales. This interest stems from the need to address design issues associated with small devices such as micro- and nanofluidics as well as from the need to understand phenomena and interactions occurring on micro-/nanometre scales. Microfluidics and nanofluidics technologies are relevant to many applications in engineering, physics, chemistry, biochemistry, nanotechnology, and biotechnology. Advances have been made in terms of practical application ranging from the design of systems allowing smaller volumes of fluids to be processed, aiming at achieving multiplexing, automation, and high-throughput screening, to on-chip characterisation.

This Special Issue was seeded as a result of a Special Session at the 2014 Conference on Micro and Nano Flows (MNF2014), held at University College London, 7-10 September, which was the fourth, successful conference of the series. The Special Session was entitled Biomedical Microfluidics of Lab-on-a-Chip Technology and was organised by the two lead editors. A unique feature of the conference series is that it provides a forum for scientists and engineers working on small scale flows in the areas of both biofluid mechanics and thermofluids engineering to present their recent research findings. This allowed for interdisciplinary exchange of ideas that advanced understanding of

Carola König

Carola.Koenig@brunel.ac.uk

1 College of Engineering, Design and Physical Sciences, Brunel University London, London, UK

2 Department of Electronics, Information and Bioengineering, Politecnico di Milano, Milan, Italy

3 Faculty of Engineering, University of Strathclyde, Glasgow, UK micro- and nanoflows and provided a platform for identifying synergies and exploring cross-over opportunities in biomedicine/biotechnology and thermofluids engineering. Such cross-over was nicely illustrated in the plenary lecture given by Professor Dimitris Drikakis entitled "Advances and Challenges in Computational Research of Micro Nano Flows" whose lecture presented application examples across a range of technology sectors. The lead paper of this issue, which presents recent advances in multiscale modelling with a focus on a variety of mesoscale and hybrid molecular-continuum methods, is based on his plenary lecture. The remaining articles in this issue were selectively invited and successfully passed the journal's high-standard peer-review process.

In the last 20 years, a key goal in the drug discovery field has been the development of reliable in vitro models. The paper by Rasponi et al. presents the development of lab on a chip (LoC) technology that allows for testing myelotoxic effects of drugs and chemicals. The LoC platform consists of 180 bioreactor chambers, designed to host a controlled and limited number of cells in suspension. The probability of each chamber to contain a colony-forming unit was evaluated based on the clonogenicity of the cell population and on a specifically developed statistical model. Kefala et al. presented a labyrinth split and merge (SAM) micromixer for bioanalytical applications. By means of a computational study, they demonstrated the mixing advantages of SAM compared to passive micromixers. Experimental validation based on fluorescence microscopy was conducted for enzymatic digestion of DNA. Ritos et al. introduced flow simulations of water through carbon nanotube membranes of realistic thickness, based on a new hybrid molecular-continuum model. The results of their model for a membrane thickness of $150 \mathrm{~nm}$ compared well with computationally more expensive molecular dynamics (MD) simulations. 
Drikakis et al. presented the behaviour of a copper-argon nanofluid confined in a nanochannel. Their MD simulations predicted a $20 \%$ increase in thermal conductivity in narrower channels compared to macroscopic cases. Cerbelli et al. presented a study on effective dispersion and separation resolution in continuous particle fractionation. Based on a $2 \mathrm{D}$ effective advection-diffusion model, they derived a closed-form expression connecting the effective transport parameters to separation resolution in order to model the transport of suspended particles. As a benchmark, they modelled particle transport in a deterministic lateral displacement device.

Biomedical Microfluidics of Lab-on-a-Chip Technology is a rapidly growing field, and the papers included in this Special Issue present only a snapshot of activities in the field. The 5th International Conference on Micro and Nano Flows will be held in Milan, Italy, at the Polytechnico di Milano between the 11 and the 14 of September 2016 (http://www.mnf2016.com/). 